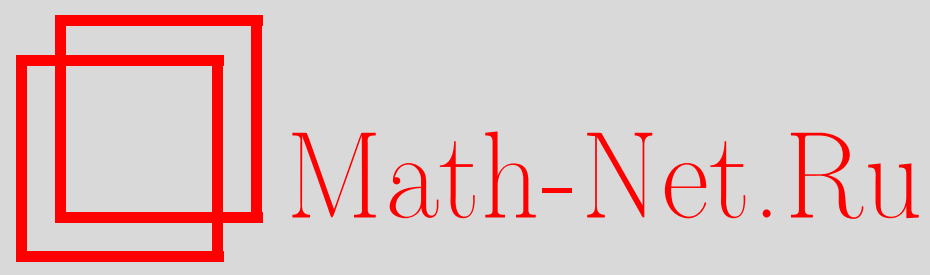

С. С. Марченков, Клоновая классификация дуально дискриминаторных алгебр с конечным носителем, Матем. заметки, 1997, том 61, выпуск 3, 359-366

DOI: https://doi.org/10.4213/mzm1510

Использование Общероссийского математического портала Math-Net.Ru подразумевает, что вы прочитали и согласны с пользовательским соглашением http://www . mathnet.ru/rus/agreement

Параметры загрузки:

IP: 54.197 .130 .99

26 апреля 2023 г., 15:51:40

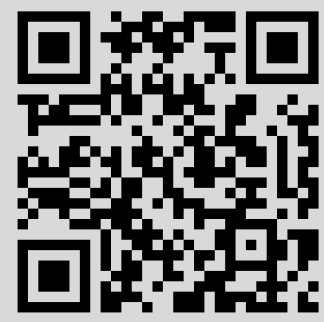




\title{
КЛОНОВАЯ КЛАССИФИКАЦИЯ ДУАЛЬНО ДИСКРИМИНАТОРНЫХ АЛГЕБР С КОНЕЧНЫМ НОСИТЕЛЕМ
}

\author{
С. С. Марченков
}

Дуально дискриминаторные алгебры рассматриваются с точностью до клонов, порождаемых операциями алгебры. В терминах двуместных отношений эффективно описьваются все клоны операций на конечном множестве, содержащие дуальный дискриминатор Пиксли. В качестве следствия определяется аналогичная клоновая классификация квазипримальных алгебр с конечньм носителем.

Библиографоия: 19 названий.

Для любого множества $E$ дуальным дискриминатором [1] на $E$ назьвается функция

$$
d(x, y, z)= \begin{cases}x, & \text { если } x=y, \\ z, & \text { если } x \neq y .\end{cases}
$$

Следуя [2], алгебру $\langle E ; F\rangle$ с носителем $E$ и множеством операций $F$ назьваем дуально дискриминаторной, если $d$ есть термальная операция в алгебре $\langle E ; F\rangle$. Алгебры $\langle E ; F\rangle,\langle E ; G\rangle$ называются әквивалентными [3], если совпадают клоны $[F],[G]$ операций, порождаемые множествами $F$ и $G$.

Дуально дискриминаторные алгебры играют заметную роль в универсальной алгебре и многозначной логике, в частности, в вопросах классификации полноты [1], [2], [4]-[9]. Например, около “половины” всех однородных алгебр [10] (в том числе с бесконечньми носителями) эквивалентны подходящим дуально дискриминаторным алгебрам конечного типа [2], [5]-[7].

Дуально дискриминаторные алгебры с конечньм носителем в [1] охарактеризованы через проективно прямоугольные подалгебры в декартовых квадратах алгебр. Однако описание из [1] неэффективно даже для алгебр конечного типа, поскольку требует проверки выполнения некоторых условий для множества всех конечноместных операций. Кроме того, как легко видеть, имеется континуальное число попарно неизоморфных дуально дискриминаторных алгебр с конечным неодноэлементным носителем. В связи с этим возникает вопрос об эффективной классификации таких алгебр.

Другая причина, побуждающая классифицировать дуально дискриминаторные алгебры, состоит в том, что всякая квазипримальная алгебра [11] является дуально дискриминаторной. Таким образом, мы можем получить одновременно и классификацию

Работа выполнена при финансовой поддержке Российского фонда фундаментальных исследований, грант № 94-01-01625. 
квазипримальных алгебр с конечным носителем. Наконец, клон $[d]$ представляет атом в решетке клонов операций на соответствующем множестве (см., например, [2]), а главный фильтр решетки, определяемый клоном $[d]$, содержит большое число важных для приложений клонов.

В данной работе мы проводим классификацию дуально дискриминаторных алгебр с конечным носителем на основе введенного отношения эквивалентности (клоновая классификация). Фактически для любого конечного множества $E$ будут описаны (теорема 1 ) все клоны операций на $E$, включающие операцию $d$. Описание эффективно и базируется на соответствии Галуа для алгебр Поста [12]. Отметим, что для фиксированного конечного множества $E$ конечность клоновой классификации вытекает из результатов работы [4]. В теореме 2 дается аналогичная классификация для квазипримальных алгебр с конечным носителем.

Для любого $k, k \geqslant 2$, в качестве стандартного $k$-элементного множества будем рассматривать множество $E_{k}=\{0,1, \ldots, k-1\}$. Совокупность всех операций на $E_{k}$ обозначим через $P_{k}$. Если $F \subseteq P_{k}$, то клон $[F]$, порожденньй множеством $F$, есть множество всех операций на $E_{k}$, термально выразимых через операции из $F$ и селекторные операции $e_{n}\left(x_{1}, \ldots, x_{i}, \ldots, x_{n}\right)=x_{i}$, где $1 \leqslant i \leqslant n, n=1,2, \ldots$ Через $L_{k}$ обозначим решетку всех клонов операций на $E_{k}$. Наименьшим элементом решетки $L_{k}$ является клон всех селекторных операций, наибольшим - клон $P_{k}$.

Наряду с операциями на $E_{k}$ будем рассматривать отношения на $E_{k}$. Множество всех отношений на $E_{k}$ обозначим через $\Pi_{k}$. Если $\rho\left(x_{1}, \ldots, x_{m}\right) \in \Pi_{k}$, то отношение $\rho$ отождествляем с множеством всех тех наборов из $E_{k}^{m}$, на которых оно истинно. В связи с этим используем термины: полное (тождественно истинное) отношение, nустое (тождественно ложное) отношение, отношение $\rho$ есть расширение $(\sigma \subseteq \rho)$ или сужение $(\rho \subseteq \sigma)$ отношения $\sigma$.

На множестве $\Pi_{k}$ определим ряд операций (см. также [12]). Конбююкиией отношений $\rho\left(x_{1}, \ldots, x_{m}\right), \sigma\left(x_{1}, \ldots, x_{n}\right)$ назовем $(m+n)$-местное отношение

$$
\rho\left(x_{1}, \ldots, x_{m}\right) \& \sigma\left(x_{m+1}, \ldots, x_{m+n}\right) .
$$

Проекцией отношения $\rho\left(x_{1}, \ldots, x_{m}\right)$ по переменной $x_{i}(1 \leqslant i \leqslant m)$ назовем $(m-1)$-местное отношение

$$
\left(\exists x_{i}\right) \rho\left(x_{1}, \ldots, x_{i}, \ldots, x_{m}\right)
$$

где областью действия квантора $\exists x_{i}$ является множество $E_{k}$. Операция перестановки и отождествления переменных предполагаем известными.

Диагоналями назьваем отношения, которые можно получить из элементарных диагоналей вида $x_{i}=x_{j}$ с помощью операций конъюнкции и отождествления переменных. Пустое отношение причисляем к диагоналям.

Пусть $R \subseteq \Pi_{k}$. Замыканием $R$ (обозначение $[R]$ ) назовем наименьшее множество отношений из $\Pi_{k}$, содержащее все диагонали и замкнутое относительно применения операций конъюнкции, проектирования, перестановки и отождествления переменных. Через $\mathscr{L}_{k}$ обозначим решетку всех замкнутых множеств отношений на $E_{k}$.

Пусть $f\left(x_{1}, \ldots, x_{n}\right) \in P_{k}, \rho\left(x_{1}, \ldots, x_{m}\right) \in \Pi_{k}$. Говорим, что операция $f$ сохраняет отношение $\rho$, если $\rho$, рассматриваемое как подмножество из $E_{k}^{m}$, образует подалгебру алгебры $\left\langle E_{k} ; f\right\rangle^{m}$. Если $F \subseteq P_{k}, R \subseteq \Pi_{k}$, то говорим, что $F$ сохраняет $R$, если каждая 
операция из $F$ сохраняет любое отношение из $R$. Множество всех операций из $P_{k}$, сохраняюших $R$, обозначим через $\mathrm{Pol} R$, а множество всех отношений из $\Pi_{k}$, сохраняемых функциями из $F,-$ через Inv $F$. Если $F$ - множество всех селекторных операций из $P_{k}$, а $R$ - множество всех диагоналей из $\Pi_{k}$, то, как нетрудно видеть, $\operatorname{Pol} R=P_{k}, \operatorname{Inv} P_{k}=R$, $\operatorname{Pol} \Pi_{k}=F, \operatorname{Inv} F=\Pi_{k}$.

Согласно теории Галуа для алгебр Поста [12] отображения Pol и Inv задают антиизоморфизм решеток $L_{k}$ и $\mathscr{L}_{k}$. Более точно, если $F \subseteq P_{k}, R \subseteq \Pi_{k}$, то $\mathrm{Pol} R-$ клон из $L_{k}, \operatorname{Inv} F-$ замкнутое множество из $\mathscr{L}_{k},[F]=\operatorname{Pol} \operatorname{Inv} F$ и $[R]=\operatorname{Inv} \operatorname{Pol} R$. Таким образом, клоны операций на $E_{k}$ можно определять через соответствующие замкнутые множества отношений на $E_{k}$.

Применим эти результаты для клоновой классификации дуально дискриминантных алгебр $\left\langle E_{k} ; F\right\rangle$. С этой целью определим, прежде всего, множество отношений Inv $[d]$.

Пусть $D_{k}$ состоит из следующих отношений на $E_{k}$ : отношения вида $x \in E$, где $E \subseteq E_{k} ;$ графики подстановок $\pi$ на $E_{k}$, т.е. отношения вида

$$
\pi\left(x_{1}\right)=x_{2}
$$

отношение

$$
x_{1}=0 \vee x_{1}=x_{2}=k-1 \text {. }
$$

Нетрудно убедиться в том, что операция $d$ сохраняет каждое отношение из $D_{k}$ (отметим, что $d$, будучи структурной однородной операцией [10], по определению сохраняет все отношения $x \in E$ и (1)). Таким образом, $[d] \subseteq \operatorname{Pol} D_{k}$.

Предположим, что $[d] \neq \operatorname{Pol} D_{k}$. Так как $D_{k}$ содержит все отношения вида (1), то клон Pol $D_{k}$ состоит из однородных операций [10]. Известно (см. [13], [14] или [15], [16] при $k=2$ и [5]-[7] при $k \geqslant 3)$, что в случае $[d] \subset \operatorname{Pol} D_{k}$ клон $\operatorname{Pol} D_{k}$ содержит хотя бы одну из следующих операций: при $k \geqslant 2$ - тернарньй дискриминатор

$$
p(x, y, z)= \begin{cases}z, & \text { если } x=y, \\ x, & \text { если } x \neq y\end{cases}
$$

при $k \geqslant 3-$ операцию

$$
l_{k}\left(x_{1}, \ldots, x_{k}\right)= \begin{cases}x_{1}, & \text { если }\left\{x_{1}, \ldots, x_{k}\right\}=E_{k} \\ x_{k} & \text { в противном случае }\end{cases}
$$

при $k \geqslant 4-$ операцию

$$
d_{k}\left(x_{1}, \ldots, x_{k-1}\right)= \begin{cases}x_{k}, & \text { если }\left\{x_{1}, \ldots, x_{k-1}, x_{k}\right\}=E_{k}, \\ d\left(x_{1}, x_{2}, x_{3}\right) & \text { в остальных случаях. }\end{cases}
$$

Проверяем, что каждая из операций $p, l_{k}, d_{k}$ не сохраняет отношения (2). Значит, $[d]=\operatorname{Pol} D_{k}$ и $\operatorname{Inv}[d]=\left[D_{k}\right]$.

Опишем теперь отношения, входящие в множество $\left[D_{k}\right]$. Покажем сначала, что множеству $\left[D_{k}\right]$ принадлежат все отношения вида

$$
x_{1}=a \vee x_{2}=b .
$$


В самом деле, если обозначить через $\rho\left(x_{1}, x_{2}\right)$ отношение $(2)$ и через $\pi$ - какую-либо подстановку на $E_{k}$, удовлетворяющую равенствам $\pi(0)=k-1, \pi(k-1)=0$, то будем иметь

$$
\left(\exists y_{1}\right)\left(\exists y_{2}\right)\left(\rho\left(y_{1}, x_{1}\right) \&\left(\pi\left(y_{1}\right)=y_{2}\right) \& \rho\left(x_{2}, y_{2}\right)\right) \equiv\left(x_{1}=k-1 \vee x_{2}=k-1\right)
$$

Обозначим последнее отношение через $\sigma\left(x_{1}, x_{2}\right)$ и возьмем такие подстановки $\pi_{1}, \pi_{2}$ на $E_{k}$, что $\pi_{1}(a)=k-1$ и $\pi_{2}(k-1)=b$. Тогда отношение $(4)$ можно задать формулой

$$
\left(\exists y_{1}\right)\left(\exists y_{2}\right)\left(\left(\pi_{1}\left(x_{1}\right)=y_{1}\right) \& \sigma\left(y_{1}, y_{2}\right) \&\left(\pi_{2}\left(y_{2}\right)=x_{2}\right)\right)
$$

ЛЕмма 1. Произвольное отношение из $\left[D_{k}\right]$ представимо в виде конбюнкиии (не обязательно с непересекающимися мнохествами переменных) одноместных отношений и двуместных отношений вида (1) или (4).

ДокАЗАТЕЛЬСТво. Проведем индукцию по построению отношений в множестве $\left[D_{k}\right]$. Замечаем, что двуместная диагональ имеет вид $(1)$, а отношение $(2)$ представимо в виде конъюнкции отношений $x_{1} \in\{0, k-1\}$ и $\left(x_{1}=0 \vee x_{2}=k-1\right)$. Поэтому для доказательства леммы достаточно показать, что требуемый в лемме вид будет иметь отношение

$$
\left(\exists x_{i}\right) \rho\left(x_{1}, \ldots, x_{i}, \ldots, x_{n}\right)
$$

где отношение $\rho$ представимо в виде конъюнкции $K$ одноместных отношений и двуместных отношений вида (1) или (4). Без ограничения общности можно считать, что $i=n$.

Пусть $\left(x_{n} \in G_{1}\right), \ldots,\left(x_{n} \in G_{s}\right)$ - все одноместные отношения из $K$, содержащие переменную $x_{n}$. Очевидно, что

$$
\left(x_{n} \in G_{1}\right) \& \cdots \&\left(x_{n} \in G_{s}\right) \equiv\left(x_{n} \in G\right)
$$

где $G=G_{1} \cap \cdots \cap G_{s}$. Если переменная $x_{n}$ в конъюнкции $K$ входит только в одноместные отношения, то отношение $\rho$ представимо в виде

$$
\rho\left(x_{1}, \ldots, x_{n}\right) \equiv\left(x_{n} \in G\right) \& \sigma\left(x_{1}, \ldots, x_{n-1}\right)
$$

где отношение $\sigma$ реализуется конъюнкцией сомножителей из $K$, не содержащих переменной $x_{n}$. Поэтому отношение $(5)$ совпадает с отношением

$$
\left(\exists x_{n}\right)\left(x_{n} \in G\right) \& \sigma\left(x_{1}, \ldots, x_{n-1}\right) .
$$

Так как первый сомножитель этого отношения либо полон (при $G \neq \varnothing)$, либо пуст (при $G=\varnothing$ ), то отношение (5) соответственно либо совпадает с отношением $\sigma$, либо пусто (пустое отношение можно представить, например, в виде $(x \in\{0\}) \&(x \in\{1\})$ ).

Пусть теперь переменная $x_{n}$ в конъюнкции $K$ входит по крайней мере в одно двуместное отношение. Рассмотрим сначала случай, когда этим отношением является отношение вида (1). Так как

$$
\left(\pi\left(x_{1}\right)=x_{2}\right) \equiv\left(x_{1}=\pi^{-1}\left(x_{2}\right)\right)
$$


где $\pi^{-1}$ - обратная к $\pi$ подстановка на $E_{k}$, то можно предполагать, что переменная $x_{n}$ входит в отношение $\pi\left(x_{j}\right)=x_{n}$. Тогда отношение $\rho$ можно представить в форме

$$
\rho\left(x_{1}, \ldots, x_{n}\right) \equiv\left(\pi\left(x_{j}\right)=x_{n}\right) \& \sigma\left(x_{1}, \ldots, x_{n}\right),
$$

где отношение $\sigma$ реализуется конъюнкцией сомножителей из $K$, отличных от $\pi\left(x_{j}\right)=x_{n}$. Замечаем, что

$$
\left(\exists x_{n}\right)\left(\left(\pi\left(x_{j}\right)=x_{n}\right) \& \sigma\left(x_{1}, \ldots, x_{n}\right)\right) \equiv \sigma\left(x_{1}, \ldots, x_{n-1}, \pi\left(x_{j}\right)\right) .
$$

Далее, легко видеть, что замена переменной $x_{n}$ на $\pi\left(x_{j}\right)$ в одноместных отношениях и в двуместных отношениях типа (1) или (4) приводит к отношениям тех же самых типов. Таким образом, отношение $\sigma\left(x_{1}, \ldots, x_{n-1}, \pi\left(x_{j}\right)\right)$ представимо в виде конъюнкции одноместных отношений и двуместных отношений вида (1) или (4).

Предположим, что конъюнкция $K$ не содержит отношений вида (1) с переменной $x_{n}$. Так как в дальнейших преобразованиях существенны лиш отношения, содержащие переменную $x_{n}$, то будем просто считать, что

$$
\rho\left(x_{1}, \ldots, x_{n}\right) \equiv\left(x_{n} \in G\right) \&\left(\bigotimes_{1 \leqslant i \leqslant n-1}\left(x_{i}=a_{i} \vee x_{n}=b_{i}\right)\right)
$$

Пусть $G=\left\{c_{1}, \ldots, c_{p}\right\}$. Тогда по определению квантора $\exists x_{n}$ отношение $\left(\exists x_{n}\right)$ $\rho\left(x_{1}, \ldots, x_{n}\right)$ совпадает с отношением

$$
\bigvee_{1 \leqslant j \leqslant p} \bigotimes_{1 \leqslant i \leqslant n-1}\left(x_{i}=a_{i} \vee c_{j}=b_{i}\right)
$$

Отношение $x_{i}=a_{i} \vee c_{j}=b_{i}$ при $c_{j}=b_{i}$ полно, а при $c_{j} \neq b_{i}$ совпадает с отношением $x_{i}=a_{i}$. Положим для любого $j, 1 \leqslant j \leqslant p$,

$$
N_{j}=\left\{i: 1 \leqslant i \leqslant n-1, c_{j}=b_{i}\right\}
$$

Тогда

$$
\left(\exists x_{n}\right) \rho\left(x_{1}, \ldots, x_{n}\right) \equiv \bigvee_{1 \leqslant j \leqslant p} \&_{i \in\{1, \ldots, n-1\} \backslash N_{j}}\left(x_{i}=a_{i}\right) .
$$

Множества $N_{1}, \ldots, N_{p}$ попарно не пересекаются, а объединение этих множеств совпадает с $\{1, \ldots, n-1\}$. Если одно из множеств $N_{1}, \ldots, N_{p}$ равно $\{1, \ldots, n-1\}$, то, очевидно, отношение $\left(\exists x_{n}\right) \rho\left(x_{1}, \ldots, x_{n}\right)$ полно. В противном случае пусть, например, $N_{1}, \ldots, N_{q}$ - все непустые и отличные от $\{1, \ldots, n-1\}$ множества из числа $N_{1}, \ldots, N_{p}$. Тогда в эквивалентности (6) вместо $p$ можно взять $q$. С использованием стандартных преобразований из алгебры логики получаем далее

$$
\bigvee_{1 \leqslant j \leqslant q} \&_{i \in\{1, \ldots, n-1\} \backslash N_{j}}\left(x_{i}=a_{i}\right) \equiv \bigotimes_{s, t}\left(x_{s}=a_{s} \vee x_{t}=a_{t}\right),
$$

где конъюнкция в правой части распространяется по всем тем значениям $s, t$ из $\{1, \ldots, n-1\}$, которые лежат в различных множествах $N_{1}, \ldots, N_{q}$. Лемма доказана. 
Лемма 2. Пусть отношение $\rho\left(x_{1}, \ldots, x_{n}\right)$ представимо в виде конбюнкции одноместных отношений и двуместных отношений типа (1) или (4). Тогда $\rho$ можно также представить в виде конбюнкиии одноместных отношений и двуместных отношений типов

$$
\begin{gathered}
\left(x_{1} \in E\right) \&\left(\pi\left(x_{1}\right)=x_{2}\right), \\
\left(x_{1} \in E\right) \&\left(x_{2} \in G\right) \&\left(x_{1}=a \vee x_{2}=b\right),
\end{gathered}
$$

причем каждое из указанных отношений входит в множество $[\rho]$.

ДокАЗАТЕЛЬСтво. Для любого $i, 1 \leqslant i \leqslant n$, через $\sigma_{i}\left(x_{i}\right)$ обозначим проекцию отношения $\rho$ по всем переменньп, отличньп от $x_{i}$. Аналогичньп образом определяем отношения $\tau_{i j}\left(x_{i}, x_{j}\right)$, где $1 \leqslant i<j \leqslant n$. Очевидно, что отношения $\sigma_{i}, \tau_{i j}$ принадлежат множеству $[\rho]$.

Из определения проекции вытекает, что отношения $\sigma_{i}, \tau_{i j}$, рассматриваемые от всех переменных $x_{1}, \ldots, x_{n}$, будут являться расширениями отношения $\rho$. Расширением $\rho$ будет являться также любая конъюнкция отношений $\sigma_{i}\left(x_{i}\right), \tau_{i j}\left(x_{i}, x_{j}\right)$.

С другой стороны, если в конъюнктивное представление отношения $\rho$ входит отношение $\sigma\left(x_{i}\right)$, то, очевидно, отношение $\sigma\left(x_{i}\right)$ может являться лишш расширением отношения $\sigma_{i}\left(x_{i}\right)$. Аналогично для отношения $\tau\left(x_{i}, x_{j}\right)$, входящего в конъюнктивное представление $\rho$, и отношения $\tau_{i j}\left(x_{i}, x_{j}\right)$. Значит, если образовать конъюнкцию $K$ всех тех отношений $\sigma_{i}\left(x_{i}\right), \tau_{i j}\left(x_{i}, x_{j}\right)$, которые отвечают входящим в конъюнктивное представление отношения $\rho$ отношениям $\sigma\left(x_{i}\right)$ и $\tau\left(x_{i}, x_{j}\right)$, то отношение $\rho$ будет являться расширением отношения $\varkappa$, реализуемого конъюнкцией $K$. Однако, как отмечалось вьше, отношение $\varkappa$ есть расширение отношения $\rho$. Следовательно, $\varkappa$ совпадает с $\rho$.

Если отношение $\tau\left(x_{i}, x_{j}\right)$, входящее в конъюнктивное представление отношения $\rho$, имеет вид (1) или (4), то соответствуюшее ему отношение $\tau_{i j}\left(x_{i}, x_{j}\right)$, будучи сужением отношения $\tau\left(x_{i}, x_{j}\right)$, может иметь лишь вид (7) или (8). Лемма доказана.

Пусть $R$-множество одно- и двуместных отношений на $E_{k}$. Будем говорить, что множество $R$ 2-замкнуто, если $R$ совпадает с множеством одно- и двуместных отношений из $[R]$.

ТЕОРемА 1. Для любого $k, k \geqslant 2$, число попарно не әквивалентных дуально дискриминаторных алгебр $\left\langle E_{k} ; F\right\rangle$ равно числу различных 2-замкнутых множеств отношений на $E_{k}$, все двуместные отношения которых имеют вид (7) или (8) либо представимы в виде конбюнкиии двух одноместных отношений. Клоны $[F]$, содержащие дуальный дискриминатор $d$, и только они представимы в виде $\mathrm{Pol} R$, где $R$ - подходящие 2-замкнутые множества отношений указанного типа.

ДоказАтЕльство. Как установлено выше, $[d]=\operatorname{Pol} D_{k} \cdot \operatorname{Tak~как~} \operatorname{Pol} D_{k}=\operatorname{Pol}\left[D_{k}\right]$ (см. [12]), то имеем также $[d]=\operatorname{Pol}\left[D_{k}\right]$.

По соответствию Галуа между решетками $L_{k}$ и $\mathscr{L}_{k}$ для всякого клона $F$ с условием $d \in F$ имеется единственное замкнутое множество отношений $R$, где $R \subseteq\left[D_{k}\right]$ и $F=\operatorname{Pol} R$. Согласно лемме 1 множество $R$ состоит из отношений, которые представимы в виде конъюнкции одноместных отношений и двуместных отношений типов (1) или (4). Обозначим через $R_{2}$ множество всех одно- и двуместных отношений из $R$. В силу леммы 2 и ввиду замкнутости множества $R$ множество $R_{2} 2$-замкнуто и $\left[R_{2}\right]=R$. 
Кроме того, по лемме 2 каждое двуместно отношение из $R_{2}$ либо представимо в виде конъюнкции двух одноместных отношений, либо имеет вид (7) или (8). Из $\left[R_{2}\right]=R$ следует (см. [12]), что $\mathrm{Pol} R_{2}=\mathrm{Pol} R$. Таким образом, $F=\operatorname{Pol} R_{2}$ и теорема доказана.

Квазипримальная алгебра может быть определена [11] как алгебра, в которой терминальной операцией является терминальньй дискриминатор (3). Так как

$$
d(x, y, z)=p(x, p(x, y, z), z)
$$

то квазипримальная алгебра является также дуально дискриминаторной.

Мы хотим провести классификацию квазипримальных алгебр с конечным носителем в духе доказанной теоремы 1 . С этой целью для любого $k, k \geqslant 2$, через $Q_{k}$ обозначим конечное множество отношений на $E_{k}$, состоящее из всех отношений вида $x \in E$, где $E \subseteq E_{k}$, и всех отношений вида (1). Легко видеть, что операция $p$ сохраняет каждое отношение из $Q_{k}$. Таким образом, $[p] \subseteq \operatorname{Pol} Q_{k}$. Так как множество $Q_{k}$ содержит все отношения (1), то клон $\operatorname{Pol} Q_{k}$ состоит только из однородньг операций на $E_{k} . \mathrm{B}[2]$ показано, что при $\mathrm{Pol} Q_{k} \neq[p]$ клон $\mathrm{Pol} Q_{k}$ обязан содержать операцию

$$
r_{k}\left(x_{1}, \ldots, x_{k-1}\right)= \begin{cases}x_{k}, & \text { если }\left\{x_{1}, \ldots, x_{k-1}, x_{k}\right\}=E_{k} \\ x_{1} & \text { в противном случае. }\end{cases}
$$

Однако, как легко видеть, операция $r_{k}$ не сохраняет отношения $x \in E_{k-1}$. Значит, на самом деле $[p]=\operatorname{Pol} Q_{k}$.

Далее действуем по той же схеме и для дуально дискриминаторньх алгебр. При этом можно воспользоваться ослабленными версиями лемм 1 и 2 (в случае квазипримальных алгебр отсутствуют отношения вида (4) или (8)). В результате мы приходим к следующему утверждению.

Теорема 2. Для любого $k, k \geqslant 2$, число попарно не әквивалентных квазипримальных алгебр $\left\langle E_{k} ; F\right\rangle$ равно числу различных 2-замкнутых множеств отношений на $E_{k}$, все двуместные отношения которых имеют вид (7) либо представимы в виде конғюнкиии двух одноместных отношений. Клоны $[F]$, содержащие тернарный дискриминатор $p, u$ только они представимы в виде $\mathrm{Pol} R$, где $R$ подходящие 2-замкнутые мнохсества отношений указанного типа.

Отметим, что в [17] указаны представители всех классов эквивалентности квазипримальных алгебр с трехэлементным носителем.

Нетрудно показать, что при $F \subseteq P_{k}$ клон $[F]$, содержащий тернарный дискриминатор $p$, является классом функций $k$-значной логики, замкнутом относительно операции суперпозиции и операции разветвления по предикату равенства [18], [19]. В [18] для любого $k, k \geqslant 2$, установлена конечность таких замкнутьх классов, а в [19] эти классы охарактеризованы в теоретико-категорных терминах. 


\section{СПИСОК ЦИТИРОВАННОЙ ЛИТЕРАТУРЫ}

[1] Fried E., Pixley A. F. The dual discriminator function in universal algebra // Acta Sci. Math. 1979. V. 41. №1-2. P. 83-100.

[2] Csákány B., Gavalcová T. Finite homogeneous algebras I // Acta Sci. Math. 1980. V. 42. № 1-2. P. 57-65.

[3] Grätzer G. Universal algebra. Princeton: Van Nostrand, 1968.

[4] Baker K. A., Pixley A. F. Polynomial interpolation and the Chinese remainder theorem for algebraic systems // Math. Z. 1975. V. 143. P. 165-174.

[5] Марченков С. С. О замкнутых классах самодвойственных функций многозначной логики // Проблемы кибернетики. 1979. №36. С. 5-22.

[6] Марченков С. С. Об однородных алгебрах // Докл. АН СССР. 1981. Т. 256. № 4. C. $787-790$.

[7] Марченков С. С. Однородные алгебры // Проблемы кибернетики. 1982. № 39. С. 85-106.

[8] Марченков С. С. О классификации алгебр со знакопеременной группой автоморфизмов // Докл. АН СССР. 1982. Т. 265. № 3. С. 533-536.

[9] Марченков С. С.Классификация алгебр со знакопеременной группой автоморфизмов // Матем. вопросы кибернетики. 1989. № 2. С. 100-122.

[10] Marczewski E. Homogeneous algebras and homogeneous operations // Fund. Math. 1964. V. 56. P. 81-103.

[11] Pixley A. F. The ternary discriminator function in universal algebra // Math. Ann. 1971. V. 191. P. 167-180.

[12] Бондарчук В. Г., Калужин Л.А., Котов В.Н., Ромов Б. А. Теория Галуа для алгебр Поста. I // Кибернетика. 1969. № 3. С. 1-10; II // Кибернетика. 1969. № 5. С. 1-9.

[13] Post E. L. Introduction to a general theory of elementary propositions // Amer. J. Math. 1921. V. 43. P. 163-185.

[14] Post E. L. Two-valued iterative systems of mathematical logic. Ann. of Math. Stud. V. 5. Princeton: Univ. Press, 1941.

[15] Яблонский С. В., Гаврилов Г. П., Кудрявцев В. Б. Функции алгебры логики и классы Поста. М.: Наука, 1966.

[16] Марченков С. С., Угольников А. Б. Замкнутые классы булевых функций. Препринт. М.: ИПМ АН СССР, 1990.

[17] Csákány B., Gavalcová T. Three-element quasi-primal algebras // Studia Sci. Math. Hungar. 1981. V. 16. P. 167-180.

[18] Тайманов В.А. О функциональных системах $k$-значной логики с операциями замыкания программного типа // Докл. АН СССР. 1983. Т. 268. №6. С. 1307-1310.

[19] Соловьев В. Д. Замкнутые классы в $k$-значной логике с операцией разветвления по предикату // Дискретная математика. 1990. Т. 2. №4. С. 19-25. 